\title{
Carnets
}

Revue électronique d'études françaises de l'APEF

Deuxième série - 6 | 2016

Exotopies de Barthes

\section{Barthes sémiologue et écrivain traduit au Portugal (1973-2015)}

\section{Dominique Faria et João da Costa Domingues}

\section{(2) OpenEdition}

Journals

\section{Édition électronique}

URL : http://journals.openedition.org/carnets/715

DOI : $10.4000 /$ carnets. 715

ISSN : 1646-7698

Éditeur

APEF

\section{Référence électronique}

Dominique Faria et João da Costa Domingues, « Barthes sémiologue et écrivain traduit au Portugal (1973-2015) », Carnets [En ligne], Deuxième série - 6 | 2016, mis en ligne le 31 janvier 2016, consulté le 30 avril 2019. URL : http://journals.openedition.org/carnets/715 ; DOI : 10.4000/carnets.715

Ce document a été généré automatiquement le 30 avril 2019.

\section{(c) (i) (8)}

Carnets est mis à disposition selon les termes de la licence Creative Commons - Atribution - Pas d'utilisation commerciale 4.0 International. 


\title{
Barthes sémiologue et écrivain traduit au Portugal (1973-2015)
}

\author{
Dominique Faria et João da Costa Domingues
}

1 Les œuvres et la pensée de Roland Barthes ne sauraient se confiner à l'hexagone ni au monde francophone. Par la traduction, elles ont traversé des frontières et intégré de nouveaux contextes de réception. Ainsi, dans la mesure où, pour citer Bakhtine, « une culture étrangère ne se révèle dans sa complétude et dans sa profondeur qu'au regard d'une autre culture » (Bakhtine, 1984 : 348), faut-il donc, pour connaître complètement et profondément Roland Barthes, envisager les regards étrangers que l'on a portés sur son travail. Cette étude vise précisément à dégager la réception de Roland Barthes dans un de ces contextes étrangers, celui du Portugal.

2 Notre corpus sera constitué par les traductions des ouvrages dont Barthes est le seul auteur, ainsi que par des textes critiques portant sur son œuvre et publiés au Portugal. L'étude comprend la période qui va de 1973 - date de la première traduction de Barthes à 2015. Notre propos est de saisir ce qu'on a lu de Barthes, comment il a été présenté au lecteur portugais, comment on a interprété ses enseignements, bref l'image que l'on a construite de Roland Barthes.

\section{Les traductions de Barthes au Portugal}

L'année 1973 constitue un des moments-charnières de l'introduction de Barthes au Portugal. Cette année-là, sont publiées pour la première fois les versions portugaises de Mythologies et Le Degré zéro de l'écriture, soit respectivement seize et vingt ans après la date de parution des versions françaises. Ce n'est sûrement pas une coïncidence que la Révolution des CEillets ait eu lieu un an plus tard. Auparavant, le Portugal vivait sous un régime politique dictatorial qui exerçait une censure extrême aux publications. Comme l'atteste la préface d'Eduardo Prado Coelho, dont nous parlerons plus en détail ci-dessous, la révolution du 25 avril 1974 apporta une liberté d'expression qui aura également un fort impact en ce qui concerne la traduction. 
Cela ne veut pas dire que Barthes était un inconnu au Portugal avant 1973. Au long de cette étude nous évoquerons plusieurs textes critiques publiés par des intellectuels portugais, qui lisaient Barthes en français, bien avant sa parution en portugais. C'est le cas d'Eduardo Prado Coelho, de José Augusto Seabra et de Maria Alzira Seixo, entre autres. L'année 1973 est néanmoins décisive car la traduction a permis d'amplifier la résonance des textes de Barthes au Portugal, au-delà des cercles des intellectuels francophiles.

5 Connaître le délai entre la date de parution des textes et leur traduction nous permet de saisir combien le travail de l'auteur a attiré les Portugais. Or, précisément, ces deux premières versions portugaises déclenchent une succession de traductions qui accompagnent de près la parution des livres en France. Ainsi, Le Plaisir du texte et Barthes par Roland Barthes sont publiés au Portugal en 1974 et en 1976 respectivement, un an seulement après leur première édition française. De même, Leçon et Le Grain de la voix sont traduits en 1979 et 1982, avec un retard de deux ans pour le premier et un an pour le second par rapport à leur parution en France. Ceci montre que l'on accompagnait de près le parcours de Barthes, et que l'on était avide d'en avoir accès en portugais. Les moyens éditoriaux étant moins développés à la fin des années 70 qu'ils ne le sont de nos jours, publier une traduction un an seulement après la première édition nous semble être assez symptomatique.

Le nombre de rééditions nous en dit également beaucoup sur l'intérêt que les écrits de Barthes ont suscité au Portugal, et ce même sans prendre en compte les chiffres des tirages. Jusqu'à présent, les livres qui ont été réédités le plus souvent sont Mythologies ( 9 éditions), Le Plaisir du texte (9 éditions), Le Degré zéro de l'écriture (7 éditions) et Fragments d'un discours amoureux (5 éditions). Remarquons aussi que c'est dans les années 70 et 80 , une période d'espoir et d'enthousiasme, après la chute de la dictature, que se concentre le plus grand nombre de rééditions, ce qui montre, à nouveau, l'enthousiasme pour le travail de Barthes pendant ces années-là au Portugal. Mythologies, par exemple, a fait l'objet de neuf éditions (en 1973, 1976, 1979, 1984, 1987, 1988, 1997, 2007 et 2012) dont 6 datent des années 70 et 80 .

7 En 1989, la plupart des ouvrages de Barthes qui sont traduits au Portugal l'étaient déjà (Maria Alzira Seixo disait, déjà en 1982, que " presque tout était traduit ; mal traduit pour la plupart $»^{1}$, mais traduit tout de même). Suit une période de 20 ans sans que de nouvelles traductions ne voient le jour, époque qui se termine en 2009 avec la publication posthume de Journal de deuil et de Carnets du voyage en Chine: le premier fut publié au Portugal l'année même de sa sortie en France et le second l'année suivante, en 2010.

8 Parmi les livres dont Barthes est le seul auteur, neuf n'ont toujours pas été traduits au Portugal : Michelet (1954), Sur Racine (1963), La Tour Eiffel (1964), L'Empire des signes (1970), Sollers écrivain (1979), Incidents (1987), Le Neutre (2002), Écrits sur le théâtre (2002) et Comment vivre ensemble (2002). Une partie de ses écrits reste donc inaccessible au public portugais qui ne lit pas le français.

9 Pour mieux comprendre la réception des textes de Barthes au Portugal il faut également tenir compte de la maison d'édition qui s'en est chargée et des choix éditoriaux par lesquels elle les a présentés au public portugais. Les traductions des ouvrages de Roland Barthes ont été publiées dans une seule maison d'édition portugaise - Edições 70 -, créée aux débuts des années 70, sous la dictature de Salazar ${ }^{2}$. Lors des premières traductions de Barthes, Edições 70 était donc une très jeune maison d'édition qui visait à introduire des 
nouveautés dans l'espace éditorial portugais. Spécialisée dans des publications du domaine des sciences humaines, Edições 70 crée une collection appelée "Signos" [Signes], dans laquelle les textes de Barthes ont été inclus, et qui est présentée sur le site de la maison d'édition comme une collection pionnière dans la diffusion des textes des grands intellectuels dans le domaine des sciences du langage et de l'analyse littéraire ${ }^{3}$ Y ont été inclus également des ouvrages de Kristeva, Chomsky, Todorov et Foucault, entre autres.

En 2007, toutefois, les Edições 70 créent une collection exclusivement consacrée à cet auteur, appelée « Obras de Roland Barthes » [๕uvres de Roland Barthes], où les textes de Barthes sont réédités. Les couvertures en ont été remaniées, portant maintenant une seule photographie de Barthes, de couleur différente pour chaque ouvrage. Il s'agit d'une photo de l'auteur dans sa trentaine, ce qui, par contraste avec une photo prise après sa consécration, évoque plutôt l'irrévérence du jeune Barthes et le caractère innovant de sa réflexion. Ces couvertures remplacent les typiques images abstraites ou les détails de tableaux célèbres qui illustraient la plupart des couvertures des ouvrages de la collection «Signos » et qui s'adaptaient mieux au domaine de la linguistique et de la sémiotique.

Dans cette nouvelle collection, la majorité des textes sont classés dans trois catégories thématiques: la linguistique (qui regroupe la plupart des textes), l'essai littéraire et la philosophie. Les exceptions en sont Critique et Vérité, classé sous «Littérature » et La Chambre claire classé sous " photographie ». Ces modifications, qui ne sont pas seulement esthétiques, montrent comment plus récemment on a bien fini par reconnaître la singularité du travail de Barthes, sa multidisciplinarité et son importance. Barthes n'est plus perçu comme le représentant de la sémiologie ou comme linguiste, mais plutôt comme un auteur à part entière.

Les textes de cette nouvelle collection ne semblent cependant pas avoir subi de rectifications importantes par rapport aux premières versions ${ }^{4}$. D'ailleurs, au Portugal, en général ces textes n'ont pas été retraduits ou revus, et les éditions portent rarement des préfaces écrites par des Portugais. Les ouvrages de Barthes surgissent donc généralement sans textes explicatifs (bien que quelques éditions soient précédées de la préface de l'éditeur français traduite). Même la présentation sur la quatrième de couverture n'est généralement qu'un extrait du livre ou alors, plus rarement, quelques phrases qui indiquent brièvement le sujet du texte.

13 Pour étudier la réception de Barthes au Portugal il faut également savoir qui ont été ses traducteurs. Or, nous constatons qu'il y a eu 15 traducteurs portugais. Certains étaient des intellectuels plus ou moins connus, soit des enseignants à l'université parmi lesquels on compte José Augusto Seabra et Maria de Santa Cruz, soit des écrivains comme Ana Mafalda Leite et Miguel Serras Pereira ou encore des journalistes comme Luis Filipe Sarmento et Tereza Coelho. D'autres étaient plutôt des traducteurs professionnels, comme Maria Margarida Barahona, Manuela Torres, Alexandre Melo ou António Gonçalves. Il n'y a donc point, au Portugal, un traducteur de Barthes spécialisé, sauf peutêtre José Augusto Seabra, mais pour d'autres raisons, comme on le verra ci-dessous.

Ces traducteurs sont, pour reprendre la notion de Lawrence Venuti (1995), presque invisibles. En effet, dans ces éditions, nous ne trouvons que très rarement des commentaires sur la traduction et l'intervention du traducteur dans le texte passe plutôt inaperçue. Le seul traducteur portugais qui ait écrit une préface à sa traduction est José Augusto Seabra. Et même lui, il ne parle de traduction qu'une seule fois, dans une note de bas de page, pour justifier un de ses choix. Qui plus est, les traductions portugaises des 
textes de Barthes portent peu de notes du traducteur ${ }^{5}$. Celles-ci sont très brèves et indiquent souvent le mot utilisé dans la version française ou signalent une situation de double sens que la traduction ne reproduit point. Cette absence de problématisation semble emblématique du manque de sensibilité qui semble s'éterniser au Portugal pour les questions de la traduction. Par conséquent, et compte tenu des choix éditoriaux, le texte de Barthes est généralement présenté au lecteur sans aucune problématisation, sans aucune mise en contexte.

\section{Comment a-t-on parlé de Barthes au Portugal ?}

\section{Quelques références par ordre chronologique ${ }^{6}$}

Lorsqu'en 1966, dans son article « Roland Barthes: Critique et vérité »" Eduardo Prado Coelho définit Barthes comme le représentant par excellence de la «nouvelle critique $»^{8}$, il rend compte, non seulement de l'intérêt que cette thématique a suscité au Portugal, mais aussi de la discussion qui s'est établie entre Barthes et Raymond Picard ${ }^{9}$, l'auteur de Nouvelle critique, nouvelle imposture (1965). Cet article d'Eduardo Prado Coelho, à l'image d'autres qui en ont parlé mais qui n'ont pas fait les gros titres des journaux, montre à l'évidence que l'intérêt pour Barthes au Portugal date de bien avant ses premières traductions en portugais.

En effet, ce n'est qu'en 1973 que paraît Mitologias ${ }^{10}$, la première traduction de Barthes. Dans sa Préface, José Augusto Seabra ${ }^{11}$, grand disciple de Barthes, dessine la trajectoire de son maître ainsi que la circulation de ses textes dans l'espace littéraire contemporain ${ }^{12}$. L'année suivante (1974) à la veille de la Révolution, une autre traduction de Barthes est publiée au Portugal, celle du Plaisir du texte, dont la préface ${ }^{13} \mathrm{~d}^{\prime} E d u a r d o$ Prado Coelho ${ }^{14}$ évoque surtout la réception de Barthes au Portugal et établit, d'emblée, une distinction entre ce qu'il appelle le "premier Barthes », c'est-à-dire le théoricien, et le «second Barthes ", celui du "plaisir du texte» (Apud Barthes, 1973: 19). Cette distinction est consolidée en 1980, lorsqu'à nouveau José Augusto Seabra publie celle qui est, nous semble-t-il, l'étude la plus étendue sur Barthes au Portugal, à savoir Poiética de Barthes (1980). Seabra, tout en reconnaissant qu'au Portugal on considère Barthes comme un théoricien (linguiste ou sémiologue), estime que Barthes est plutôt un écrivain.

Par ailleurs, en 1982, deux ans après la mort de Barthes, un numéro de la revue Nova Renascença lui consacre toute une section, intitulée "Hommage à Roland Barthes", comprenant plusieurs textes en français ${ }^{15}$ et deux en portugais : dans le premier, Norma Backes Tasca reprend et développe la sémiologie selon Roland Barthes; dans le second, Seabra s'interroge sur le rôle du mythe d'Orphée chez Barthes et souligne l'existence d'un Barthes linguiste d'abord, puis d'un Barthes sémiologue, qui débouchent finalement sur un Barthes écrivain, pensée dorénavant récurrente à propos de Barthes. Douze ans plus tard (en 1994), cette même revue consacre un nouveau numéro entièrement à Barthes. Dans son introduction intitulée "Roland Barthes revisité " ${ }^{16}$, on revient sur la réception de Barthes depuis sa mort, et on observe que, si dans les milieux universitaires on avait continué à étudier Barthes, certains intellectuels l'avaient déjà oublié, voire rejeté. On y signale cependant une « renaissance de Barthes » dès le début des années 90, notamment à l'occasion de l'édition des Euvres complètes de Roland Barthes. Dans ce volume, on retiendra l'article de Alzira Seixo qui, tout en évoquant le grand intérêt que le travail de Barthes a suscité - au Portugal et ailleurs - dans les années 70, propose, avec cette 
distance, de le repenser en termes de promoteur de l'idée d'une science de la littérature, étant donné ses apports à l'étude de ce domaine.

Plus de quinze ans plus tard, en 2009, dans la revue Colóquio Letras, Jean-Claude Pinson en traduction portugaise - fusionne les différentes approches et convoque la figure emblématique de l'écrivant-penseur qui se révèle dans les "Leçons" au Collège de France, et qui n'est plus (seulement) le théoricien des Essais critiques. Dans ses « Leçons », Barthes serait dans un ailleurs, en train d'inventer une nouvelle image de l'écrivain, dans "l'arrière-garde de l'avant-garde", comme il aimait à se définir, d'un écrivain des frontières, des territoires limitrophes où les identités s'échangent, où se mêlent les genres. Pour Barthes, selon Pinson, l'idéal serait de créer un texte qui ne serait qu'une partition musicale de mots et de phrases (Pinson, 2009: 18), et la nouvelle Science de la littérature devrait désormais prendre " pour objet, non pas les sens pleins de l'œuvre, mais plutôt le sens vide qui les supporte tous ». (apud Júdice, 2009 : 29).

Pour conclure cet aperçu de l'introduction de Barthes parmi nous, nous mettrions en exergue ce que disait, déjà en 1982, Maria Alzira Seixo, lorsqu'elle affirmait que Barthes était très présent dans la culture portugaise ${ }^{17}$ et que son œuvre était vue comme inspiratrice de découvertes, source d'émerveillements et d'hypothèses de développements à la fois littéraires et spéculatifs d'un certain parcours spirituel, ainsi que d'un goût pour la lecture qui va de la nouvelle critique à la sémiologie et du structuralisme à la théorie du texte (cf. Seixo, 1982: 14). Cette réflexion montre, s'il le fallait, que Barthes s'inscrit d'emblée au Portugal dans une lecture/réception protéiforme.

\section{Mais qu'avait-on lu de Barthes, vraiment ? Et qu'avait-on retenu de sa pensée?}

\subsection{Un premier élément de réponse}

Un premier élément de réponse nous est fourni dans la préface de la traduction de mythologies, ou Seabra présente, en 1973, le travail de Barthes de la manière suivante

a. Son œuvre est d'abord un work in progress autour de la notion théorique et critique de l'écriture qui est elle-même avant tout une pratique de l'écriture ${ }^{18}$.

b. Ensuite, dans Mythologies, précise-t-il, il est surtout question de la notion de signification et de la valeur connotative de la littérature : le discours de l'écrivain dit ce qu'il dit, mais il dit aussi que c'est littérature. Chez Barthes, l'écriture se trouve interpénétrée de deux formes de langage, au sein desquelles il se déplace: le métalangage (du critique littéraire) et le langage-objet (langage littéraire, de l'écrivain) qui est un langage de connotation (cf. Mitologias, 1973 : XVI) ${ }^{19}$.

c. Mais avec sa défense du théâtre de Brecht - qui se présente comme une anti-physis, une antinature -, il revient, tout formaliste qu'il est, à la dimension idéologique et politique de la littérature : une littérature qui produit un message de signification, à savoir « un procès qui produit le sens et non le sens lui-même » (Barthes, $1981: 260)^{20}$.

d. Enfin, Seabra y réitère l'idée d'un Barthes écrivain avant tout. Il reconnaît que «[j]usqu'à présent et par contagion d'importation, ce qui compte parmi nous c'est le théoricien (le linguiste, le sémiologue) ou Barthes critique », mais il ajoute: pour moi, «les deux se fondent dans Barthes écrivain » (in Mitologias, 1973 : xI).

21 À l'image de Seabra, d'autres tiennent Barthes pour la «figure majeure de la contemporanéité", "grand interpellateur de notre temps», grand "éveilleur de 
consciences ", "Barthes destructeur de mythes ", enfin «iconoclaste ». C'est ainsi du moins qu'il a été évoqué dans l'ouverture du Colloque de Lisbonne en 1982 où l'on a affirmé :

Barthes représente un antidote actuel et actif, toujours nécessaire à la culture officielle porteuse de stratification (...), antidote qui n'a jamais cessé d'exister à travers les temps, s'opposant à l'université, que ces interpellateurs s'appellent Abélard, Rabelais, Montaigne Rousseau, Verney (...) (AAVV, $1982: 23$ )

En somme, force est de constater que, depuis les essais sémiologiques jusqu'aux théorisations du " plaisir du texte ", dans la provocation et l'irrévérence par rapport à la culture établie, la remise en question du langage, de la littérature, de la signification, voire de tous les acquis, Barthes a toujours trouvé parmi nous son public ${ }^{21}$.

\subsection{Un deuxième élément de réponse}

Un deuxième élément de réponse nous est livré par Maria Alzira Seixo, dans la préface de Leituras de Roland Barthes (1982).

Tout en présentant les réflexions faites lors du colloque réalisé la même année à Lisbonne, elle y affirme que ce volume veut juger de l'importance de Roland Barthes pour le public portugais, et faire le point sur la compréhension, l'assimilation ou la perception de Barthes par ses lecteurs/critiques ${ }^{22}$. Une lecture, même superficielle, nous permet de détacher quelques exemples de noms et d'interprétations très significatifs :

a) Pour Crabbé Rocha (1982:25-26), Barthes transforme son métalangage en langage tout en lui conférant en permanence une touche de créativité, il se fait l'héritier d'une longue tradition, mais ose la rénover au lieu de la stratifier ${ }^{23}$. Mais, si pour Crabbé Rocha on est en face d'un "démiurge recréateur ", d'autres mettent déjà l'accent sur une pensée « inclassifiable, aérienne, fluide » qui passera toujours à travers les maillons de tout filet où l'on voudrait l'enfermer ${ }^{24}$. D'autres encore, se rappelant la conférence du Maître à Lisbonne en 1963, se souviennent du critique littéraire pour qui « l'œuvre littéraire serait comme un vaisseau porté par la mer, l'histoire " et la littérature un système de significations où le signifiant serait très fort et les signifiés très mobiles.

b) Pour Ofélia Paiva Monteiro, la meilleure définition de la place occupée par le critiqueécrivain et grand prêcheur du principe du " plaisir du texte ", est celle de "lecteur " alors que, pour Carlos Reis, c'est plutôt la question de l'intertextualité et du discours de l'idéologie qui est au cœur de la pensée barthésienne. Il précise notamment que cette intertextualité fait que l'acte d'écrire est énoncé chez lui, dès ses premiers écrits, comme un processus dynamique et intertextuel, et non comme un monologue, et que la pression de l'Histoire et de la Tradition, dont parlait Barthes lui-même, serait en quelque sorte présente dans la spécificité du discours littéraire ${ }^{26}$ (cf. 1982 : 50).

c) Luís Miguel Nava, en se proposant de parler de Barthes romancier, touche à un autre volet de l'œuvre de Barthes et réfléchit, en se référant au scepticisme de Barthes, sur l'existence possible d'une science de la littérature ${ }^{27}$. Il revient notamment sur la tentation barthésienne "d'écrire quelque chose qui pourrait s'apparenter au roman ${ }^{28}$, révélant que l'auteur lui-même nous dévoile, assez explicitement d'ailleurs, son envie, dans cette nouvelle phase de son travail, "d'essayer des formes romanesques, dont aucune ne prendrait le nom de 'roman', mais dont chacune garderait, si possible en le renouvelant, 
celui d"essai'«(cit. par Nava, in 1982: 202), se métamorphosant ainsi en essayisteécrivain.

d) Dans le même sillage, Cristina Almeida Ribeiro ${ }^{29}$ (cf. $1982:$ 267-272) se demande si Barthes pouvait aussi être considéré comme un La Bruyère du $x^{e}$ siècle. En effet, en lecteur très attentif de La Bruyère, Barthes pratique l'écriture brève, concise, voire fragmentaire, dont le plaisir émerge de l'éternel recommencement et dont l'idéal consiste - c'est Barthes qui le dit - dans la grande condensation, non de pensée, de sagesse ou de vérité, mais de musique ${ }^{30}$. (cf. Mitologias, 1976 : 112-115)

e) Par ailleurs, dans le domaine des idées, et dans une tentative de définition de l'esprit de Barthes, Pierre Jourdan observe que Barthes est « l'envers de M. Teste, de Paul Valéry »" De fait, pour le grand public des étudiants français des années 68, quand il était « interdit d'interdire », Mr. Teste ressemblerait à une métaphore du pouvoir fasciste, au moment même où Barthes apprenait, à l'école des Hautes Études à déchiffrer le langage de l'ordre, certes, mais pour s'en libérer. (cf. 1982 : 273-278)

31 f) Enfin nous détacherons une dernière idée concernant une éventuelle pensée philosophique chez Barthes, développée par José Andrade. Selon ce dernier, Barthes érige sa pensée contre la domination du sens, et surgit comme le penseur de la suspension ; c'est la victoire du lecteur contre le texte qu'il reprend selon son propre rythme, ses envies et sa méthode. Il ne s'agit donc pas de savoir ce que le texte ou son auteur veulent dire, de chercher un sens intrinsèque au texte, mais de savoir plutôt ce que moi, lecteur, je peux échanger avec cet objet insolite irrécupérable. (cf. José Andrade, in 1982 : 218) Or, en philosophie, on ne produit des mots que dans la mesure où ils sont indissociables de leur rôle de signification; c'est pourquoi le texte philosophique fuit l'inadéquation du langage au réel. Par conséquent, lire le tissu philosophique à travers le prisme de Barthes serait un acte de perversion.

\section{Conclusion}

Les traductions de Barthes au Portugal n'ont pas été immédiates; elles commencent avec un recul d'une vingtaine d'années par rapport aux parutions françaises, précisément à la veille de la Révolution de 1974, qui met fin à un régime dictatorial au Portugal. L'enthousiasme qui s'empare alors de ceux qui avaient subi la censure de Salazar a sûrement contribué à ce que, à la fin des années 70 , début des années 80 , on assiste à un vif intérêt pour les enseignements de cet auteur au Portugal. Par conséquent, dès ces années-là les traductions se succèdent suivant de très près les dates de publication des versions françaises.

Les études critiques témoignent aussi de cet enthousiasme. Au début des années 80 , après la mort de Barthes, des études cruciales sont publiées : Poiética de Roland Barthes (1980), Leituras de Roland Barthes (1982), le numéro de Nova Renascença « Hommage à Roland Barthes " (1982), ainsi que bon nombre de comptes rendus publiés dans des revues portugaises. Leurs auteurs sont unanimes lorsqu'ils attestent explicitement de l'attention portée sur le travail de Barthes au Portugal.

Après une période d'une certaine réduction du nombre de traductions, il semble qu'il y ait eu un renouveau dans cet intérêt pour Barthes entre 2007 et 2010. Les choix éditoriaux suggèrent d'ailleurs que, si entre les années soixante-dix et quatre-vingt, Barthes était perçu au Portugal comme un linguiste ou comme le représentant de la sémiologie, de nos 
jours il est plutôt perçu comme un auteur à part entière, avec une œuvre complexe et plurielle.

Les études critiques publiées au Portugal font souvent écho de cette conception. Les auteurs distinguent généralement un Barthes linguiste, un Barthes sémiologue et finalement un Barthes écrivain, dans lequel les autres se fondent. Parmi les critiques portugais, José Augusto Seabra a joué un rôle essentiel. Ancien disciple de Barthes, il s'attache à étudier et à faire connaître dans son pays l'œuvre de son maître et insiste sur cette idée que Barthes est avant tout - ou après tout - un écrivain. La pensée de Barthes a été accueillie, refoulée, parfois peut-être maltraitée ou mal comprise ; mais qui pourrait en parler de façon assertive quand Barthes ${ }^{32}$ lui-même assure que le texte, chaque texte, dit ce qu'il dit plus tout ce qu'entendent les lecteurs, qu'il n'y a pas de sens du texte, que le texte est plutôt le sens vide de tous les sens? De plus, il affirme que «[c]omme institution, l'auteur est mort», tout en ajoutant que « dans le texte, d'une certaine façon, je désire l'auteur : j'ai besoin de sa figure » (Barthes, 1973b : 45-46). L'écrivant, critique et écrivain à la fois, on l'a admiré, suivi et perpétué dans des œuvres qui marquent, encore de nos jours, un versant significatif dans les études littéraires au Portugal. Toutes ses traductions et études critiques en disent ce qu'ils disent, mais aussi tout ce que pensent et parviendront à penser ceux qui les lisent ; car, en faisant de la critique, nous ne faisons pas de la traduction, mais juste de la périphrase (cf. Barthes, 1966 : 72) que l'on propose et dont chacun dorénavant dispose ${ }^{33}$. Et s'il nous faut un mot pour clore ce parcours sur Barthes au Portugal, que ce soit un mot de Barthes luimême qui résume son chemin d'écrivant-écrivain, critique et créateur : il y a d'abord un âge où l'on enseigne ce que l'on sait; puis un moment pour la recherche, et enfin une sorte de finition et de sédimentation des savoirs appelée sapientia, c'est-à-dire "aucun pouvoir, un peu de savoir, un peu de sagesse, et un maximum de goût. » (apud Júdice, $2009: 32)$

\section{BIBLIOGRAPHIE}

BAKHTINE, Mikhaïl (1984). Esthétique de la création verbale. Paris : Gallimard.

BARTHES, Roland (1953). Le Degré zéro de l'écriture. Paris : Seuil.

BARTHES, Roland (1954). Michelet par lui-même. Paris : Seuil.

BARTHES, Roland (1957). Mythologies. Paris : Seuil.

BARTHES, Roland (1963). Sur Racine. Paris : Seuil.

BARTHES, Roland (1966). Critique et vérité. Paris : Seuil, « Le Point ».

BARTHES, Roland (1970). S/Z. Paris : Seuil.

BARTHES, Roland (1973). Le Plaisir du texte. Paris : Seuil.

BARTHES, Roland (1981). Essais critiques. Paris : Seuil. 
BARTHES, Roland (1982). Critiques III. Paris : Seuil.

<URL: http://www.edicoes70.pt/site/node/3?col=33>

PICARD, Raymond (1965). Nouvelle Critique ou nouvelle imposture. Paris : J.-J. Pauvert, « Tel quel ».

VENUTI, Lawrence (1995). The translator's invisibility. Londres, Nova Iorque : Routledge.

\section{Textes de Roland Barthes traduits en portugais, présentés par ordre chronologique de parution}

(1973a) Mitologias. trad. et préface José Augusto Seabra. Lisboa : Edições 70 (réimpr. 1976, 1979, 1984, 1987 (ed. Círculo de leitores), 1988, 1997, 2007, 2012).

(1973b) O Grau Zero da Escrita. trad. Maria Margarida Barahona. Lisboa : Edições 70 (réimpr. 1977, 1981 (O Grau Zero da Escrita et Elementos de Semiologia), 1984, 1989, 1997, 2006).

(1973c) O Prazer do Texto. trad. Maria Margarida Barahona et Préface de Eduardo Prado Coelho. Lisboa : Edições 70 (réimpr. 1974, 1976, 1980, 1983, 1988, 1997, 2001, 2009 (retraduction de M. M. Barahona et Luís Filipe Sarmento, avec en plus le texte Variações sobre a escrita).

(1975) Barthes por Barthes. trad. Jorge Constante Pereira et Isabel Gonçalves. Lisboa : Edições 70 (réimpr. 2009).

(1977) Ensaios Críticos. trad. António Massano et Isabel Pascoal, Lisboa : Edições 70 (réimpr. 2009).

(1978) Crítica e Verdade. trad. Madalena da Cruz Ferreira, Lisboa : Edições 70 (réimpres.1987, 1997, 2007).

(1979a) Lição. trad. Ana Mafalda Leite, Lisboa : Edições 70 (réimpr. 1988, 2007).

(1979b) Sade, Fourier, Loyola. trad. Maria de Santa Cruz, Lisboa : Edições 70.

(1980) S/Z. trad. Maria de Santa Cruz, Ana Mafalda Leite, Lisboa : Edições 70 (réimp. 1999).

(1981a) Fragmentos de um Discurso Amoroso. trad. Isabel Pascoal, Lisboa : Edições 70 (réimpr. 1982, 1987, 2006, 2010).

(1981b) O sistema da moda. trad. Maria de Santa Cruz, Lisboa : Edições 70.

(1981c) A Câmara Clara. trad. Manuela Torres, Lisboa : Edições 70 (réimpr. 2006, 2010).

(1982) Grão da Voz. trad. Teresa Meneses, Alexandre Melo, Lisboa : Edições 70.

(1987) Aventura Semiológica. trad. Maria de Santa Cruz, Lisboa : Edições 70.

(1987a) O Rumor da Língua. trad. António Gonçalves, Lisboa : Edições 70.

(1987b) Incidentes. trad. Tereza Coelho, Alexandre Melo, Lisboa : Edições Quetzal.

(1984) Elementos da Semiologia. trad. Maria Margarida Barahona, Lisboa : Edições 70 (réimpr. 1989, 1997, 2007).

(2009a) O óbvio e o obtuso. trad. Isabel Pascoal, Lisboa : Edições 70 (réimpr. 2014, 2015).

(2009b) Diário de luto : 26 de outubro de 1977-15 de setembro de 1979. trad Miguel Serras Pereira, Lisboa : Edições 70.

(2010) Cadernos da viagem à China. trad. Luis Filipe Sarmento, Lisboa : Edições 70. 


\section{Quelques études sur Barthes présentées par ordre chronologique de publication au Portugal}

(1973) CALVET, Louis-Jean $(1973,1996)$. Roland Barthes : um olhar político sobre o signo. trad. Adriano

D. Rodrigues, Introd. Pirouz Eftekhari. Lisboa : Editorial Vega.

(1976) BRECHON, Robert (1976). « Barthes et son double », Revista Colóquio/Letras, Ensaio, n. 32, 1976, pp. 26-30.

(1978) SEABRA, José Augusto (1978). « Nas margens de um discurso amoroso », Revista Colóquio/

Letras, Ensaio, n.․ 45, pp. 5-9.

(1978) LOSA, Margarida (1978). «A desumanização da arte e da crítica : extrapolações a partir de Ortega, Lukács, Barthes e Marcuse », Revista Colóquio/Letras, Ensaio, n.ำ46, pp. 11-19.

(1980) SEIXo, Maria Alzira (1980). « À memória de Roland Barthes, projecção de um diálogo com a ausência », Revista Colóquio/Letras, Notas e Comentários, n.ํ5, pp. 63-64.

(1980) SEABRA, José Augusto (1980). Poiética de Barthes. Porto : Brasília.

(1982) AAVV (1982). Leituras de Roland Barthes, Comunicações apresentadas ao Colóquio Barthes Fac. de Letras de Lisboa, 18 e 19 de março de 1982. Lisboa : Edições Dom Quixote.

(1982) Nova Renascença, ํ6 - vol II, Inverno 1982, « Homenagem a Roland Barthes ».

(1982) SEIXo, Maria Alzira (1982). «Recensão crítica a Poiética de Barthes, de José Augusto Seabra », Revista Colóquio/Letras. Recensões Críticas, n. 67, pp. 100-101.

(1988) Sото, Luís G. (1988). Outramente Barthes. Introd. José Augusto Seabra. Porto : Edições Nova Renascença.

(1994) Nova Renascença, Porto : Associação Cultural Nova Renascença (ce volume est entièrement consacré à R. Barthes).

(2009) JÚDICE, Nuno (2009). « Barthes e o mundo como texto », Revista Colóquio/Letras, Ensaio, n. 172, pp. 25-32.

(2009) MARQUES, Ricardo (2009). « La Mort(e) c'est moi (Sobre 'Journal de deuil' de Roland Barthes) », Revista Colóquio/Letras, Ensaio, n.ำ172, pp. 33-37.

(2009) PINSon, Jean-Claude (2009). « Barthes 'po-eta’ « , Revista Colóquio/Letras, Ensaio, n.․172, pp. 13-24.

\section{NOTES}

1. «Mas índices de ordem vária existem disseminados pelo campo da cultura portuguesa actual que nos permitem supor uma acção, talvez difusa mas nem por isso menos insistente, dos escritos de Roland Barthes entre nós: num país onde a quase totalidade da sua obra se encontra traduzida (embora, é importante repeti-lo, em grande parte mal traduzida...), onde o seu nome ocorre constantemente em estudos e publicações diversas (...)» (Seixo, 1989: 13). [Mais des indices de différents genres sont disséminés dans le champ de la culture portugaise contemporaine qui suggèrent une action, peut-être diffuse mais néanmoins insistante, des textes de Roland Barthes parmi nous : dans un pays où la presque totalité de son œuvre a été traduite (bien que, il faut le 
répéter, pour la plupart mal traduite...), où son nom surgit constamment dans des études et des publications diversifiées].

2. L'exception en est Incidents, publié chez Quetzal, en 1987.

3. Voir <URL : http://www.edicoes70.pt/site/node/3?col=33>

4. Sauf de très rares exceptions, comme la nouvelle édition de Le Plaisir du texte, de 2009, à laquelle on a ajouté le texte " Variations sur l'écriture », ainsi qu'une préface de Carlos Ossola.

5. L'exception en est la version portugaise de Le système de la mode, où abondent les notes sur la traduction. La traductrice a entrepris un travail de recherche dans les revues de mode portugaises pour retrouver les termes portugais correspondants.

6. Dans l'impossibilité de redire ici tout ce qui a été dit et écrit sur Barthes au Portugal, même en résumé, notre approche ne cible que les moments et les textes qui nous semblent les plus marquants de cette réception.

7. Le titre en portugais est " Roland Barthes : crítica e verdade ». Cet article fut publié dans un journal portugais et repris plus tard dans un recueil de textes daté de 1972.

8. Prado Coelho pensait certainement aux orientations novatrices et polémiques exposées dans l'essai de Roland Barthes Sur Racine, en 1963, où il tend à supplanter l'histoire littéraire comme méthode principale d'interprétation du texte littéraire par l'adoption de méthodes tirées du structuralisme, voulant affirmer que « le temps des œuvres » est indépendant du « temps de l'histoire ».

9. Raymond Picard (1917-1975) s'est toujours opposé à Roland Barthes considérant la « nouvelle critique » comme " des impostures ». Il est notamment l'auteur d'un ouvrage intitulé Nouvelle critique ou nouvelle imposture, coll. "Libertés ", J.-J. Pauvert, 1965. Barthes lui répondra l'année suivante avec Critique et vérité (1966). Prado Coelho y fait aussi référence à un article de Jacqueline Piattier, publié dans Le Monde, et qui aurait été reproduit dans le journal portugais Diário de Lisboa ainsi qu'à un compte rendu d'Alexandre Pinheiro Torres publié dans Seara Nova. Diário de Lisboa est un journal quotidien portugais publié entre le 7 avril 1921 et le 30 novembre 1990. Seara Nova fut créé en 1921 par un ensemble d'intellectuels portugais qui voulaient en faire une publication doctrinaire et critique, voulant ainsi éviter l'isolement de l'élite intellectuelle portugaise. Le groupe de Seara Nova, sous la dictature de Salazar (depuis 1926) et malgré la censure et les difficultés financières, a été très actif dans le combat idéologique. Avec des interventions dans le domaine littéraire et dans l'éducation, Seara Nova est aussi devenue le plus important périodique d'opposition démocratique contre le régime d'oliveira Salazar. Avec la rénovation politique et culturelle de la gauche portugaise, depuis les années soixante et jusqu'à la chute de la dictature et à la réorganisation de la vie politique et culturelle du pays, l'action de Seara Nova s'est révélée un des plus importants moteurs de consolidation de l'opposition démocratique au Portugal.

10. La traduction a été faite à Paris.

11. Il avait fait sa thèse de doctorat à Paris, sur Fernando Pessoa, sous l'orientation de Barthes.

12. Ce texte a joué un rôle décisif dans la réception de Barthes au Portugal, car il était cité par quiconque parlait de lui au Portugal. Augusto Seabra y présente son auteur et livre au lecteur portugais les repères essentiels à la compréhension de la pensée barthésienne.

13. Cette préface a la particularité d'avoir été écrite 14 jours après la révolution portugaise de 1974. Il s'agit donc d'un texte très daté où Prado Coelho, visiblement bouleversé par les événements tout récents, parle surtout de l'importance du langage, de l'interdiction d'utiliser certains mots pendant le régime dictatorial et du plaisir de pouvoir enfin les prononcer librement, et les écrire.

14. Il n'en est pas le traducteur.

15. Ces textes sont de François Wahl et de Julia Kristeva, portant surtout sur l'amitié et la douleur de la mort et de la perte.

16. L'introduction est signée par « l'équipe de rédaction » de la revue. 
17. Il y avait donc une présence et une action, bien qu'indirectes, des écrits de cet auteur parmi nous : à cette date, non seulement son œuvre était presque toute traduite en portugais, mais son nom revenait en permanence dans les études et les publications, jusque dans le domaine du journalisme.

18. Et il explique que, dès 1953, avec Le Degré zéro de l'écriture, Barthes introduit en plein centre de la problématique littéraire la notion "d'écriture ». L'écriture à l'état pur, le degré zéro de l'écriture, serait innocente, purement indicative ; par cette écriture, l'auteur serait libéré des idéologies. Mais ce n'est qu'une utopie cette littérature faite d'un « langage rêvé dont la fraîcheur (...) figurerait la perfection d'un nouveau monde adamique où le langage ne serait plus aliéné » (Barthes, $1953: 126$ ).

19. Dans Michelet par lui-même, Barthes essaie une méthode d'analyse thématique, synchronique, structurale : trouver la structure d'une existence, une thématique. Avec l'éloge à Robbe-Grillet, il met en relief cette écriture dite " objective ", sans épaisseur et qui ne suppose pas de dimension profonde. Une écriture littérale, libérée du réel, qui crée quelque chose qui se donne, l'être là, un peu comme le dasein heideggérien.

20. Quoi qu'il en soit, Mythologies, c'est Barthes qui le dit ouvertement, est une critique idéologique des mythes de la vie quotidienne française, et tout particulièrement de cette « espèce de monstre » qui est, pour lui, la petite bourgeoisie: son but était de " rendre compte des subterfuges et de dénoncer la mystification qui transforme la culture petite-bourgeoise en nature universelle ». (Interview à Tel Quel, 1947 : 96).

21. Bien qu'en simple note de bas de page, Maria Alzira Seixo ne remarque pas moins en 1982 que la commission du Colloque sur Barthes à Lisbonne cette année-là avait reçu des lettres qui sollicitaient la réalisation de ce même colloque dans d'autres endroits au Portugal, notamment dans le Nord et en Algarve (cf. 1982 : 12, note 1). Sur l'importance de Barthes au Portugal, Maria Alzira Seixo, toujours dans la même préface, le considère dès 1982 comme « une figure vraiment riche de la pensée littéraire et sociale, un écrivain aimé et un orienteur fécond » (Seixo, 1982: 13), ayant orienté plusieurs thèses de chercheurs portugais qui avaient suivi ses cours à Paris, parmi lesquels J. A. Seabra qui fut peut-être son plus grand disciple au Portugal.

22. Le public de ce colloque, dit Maria Alzira Seixo, était composé surtout d'étudiants universitaires de philosophie et de lettres ; mais il y avait aussi dans la salle « des professeurs du secondaire, fonctionnaires, psychologues, bibliothécaires, designers, journalistes, acteurs, écrivains, ingénieurs et traducteurs. Quant aux conférenciers, ils étaient presque tous des universitaires. " (in Leituras de R. Barthes, 1982 : 11-12). Outre les thèses, les colloques et les publications, d'autres indices nous permettent de croire que, aussi bien dans le domaine de la philosophie et des Lettres que dans la culture portugaise en général, une infiltration féconde de sa pensée s'est produite, et très concrètement dans le domaine de l'utilisation d'un vocabulaire sémiologique barthésien.

23. Elle parle d'un Barthes héritier d'une culture philosophique, littéraire et artistique consacrée, qu'il ne renie pas, mais qui, « comme en contrepoint, prête une attention critique permanente à des faits qui remplissent notre quotidien » (1982: 26) : qu'il parle du tour de France, de la mode, de la photographie des détergents ou de la politique, dit-elle, c'est dans ce pouvoir d'appropriation personnelle d'une culture inter-textuellement sédimentée que réside la force active de la présence barthésienne.

24. «Une œuvre et une pensée qui a des ruptures, des hiatus, volontairement éparpillées, disjointes, pour éviter toute tentation de construction d'un appareil - encore un - linguistique, sociologique ou politique. Toute son œuvre ne sert qu'à contrarier les évidences. C'est une pensée qui, selon Jacques Verclytte, insiste à refuser les clivages opérés en occident 'qui ont placé à l'opposé l'un de l'autre l'esprit et la lettre, la forme et le fond, la raison et le produit des sens'. » (1982: 28). 
25. En effet, précise Ofélia Monteiro, il a su comme personne développer cette activité dynamique d'écoute du jeu des signes que construit une œuvre littéraire. Barthes, respectant toujours la configuration historique des textes classiques français, dit-elle, et sans supprimer de sa réflexion critique une perspective historico-idéologique, nous les a pourtant réaccordés à notre langage, tout en nous séduisant par ce trait singulier qui consiste à unir la dimension métalinguistique propre à toutes les études critiques, avec la dimension connotative du discours d'un « moi-artiste ». (cf. Monteiro, $1982: 254$ )

26. Et de son écriture même, ne dit-il pas avoir « (...) pilé, pressé ensemble des idées venues de [sa] culture, c'est-à-dire du discours des autres » et avoir " continument pris appui sur ce qui s'énonçait autour de lui »? (Barthes, 1970, dans la quatrième de couverture) Or cela veut dire aussi, remarque Carlos Reis, qu'il n'y a pas de production de discours séparé de la dialectique qui s'établit entre le livre arbitre du sujet écrivant et sa soumission à tout ce qui est au-delà du texte et qui le domine ; le texte le respecte et le dépasse à la fois, car « la littérature n'est pas un objet intemporel, mais un ensemble de pratiques et de valeurs situés dans une certaine société » (Barthes et alii, Escrever... Para quê ? Para quem ?, ed. 70, 1975 : 10, apud Carlos Reis, in Barthes, $1982: 52)$ et par conséquent aussi marquée, articulée avec le système idéologique dans lequel elle a été créée.

27. Pour Barthes, le problème essentiel, jamais résolu, est le discours de la science qui pour lui ne serait qu'un, à savoir le discours algorithmique; d'où la tentation de mettre le texte littéraire en équations logiques, de recourir à des formulations, exprimant de pures relations. Mais dès qu'on utilise un idiome, on ne peut échapper à la dimension culturelle, aux connotations, aux sens multiples et dès lors il s'avère nécessaire revendiquer en faveur d'une certaine subjectivité : Barthes parle de « théoriser l'affect comme moteur de la critique » et d'instituer «une sorte d'isomorphisme entre la langue de la littérature et le discours sur la littérature ». « (...) j'ai abandonné radicalement, dit-il dès 1970 (S/Z), le discours dit critique pour entrer dans un discours de la lecture, une écriture-lecture. » (cit. por Nava, in Barthes, 1982 : 191-192).

28. R. Barthes avoue en avoir envie " pour accéder à un genre d'écriture qui ne soit plus fragmentaire » (cit. par Nava, in Barthes, 1982 : 199). Et Robbe-Grillet reconnaît que Barthes « commençait à se parler, lui, d'une façon non pas rigoureuse, (...), mais flottante, et que le romancier Barthes commençait déjà à se développer dans ses textes » (cit. par Nava, in Barthes, 1982 : 201-202).

29. Parler des Mythologies de Barthes quand on sait que son auteur a entrepris après des chemins si divers, semblerait insister sur une image dépassée de l'auteur et par conséquent inutile. Et pourtant, il faut remarquer que, avec Le Degré zéro de l'écriture, ce texte a marqué le premier parcours barthésien qui mérite toute notre attention.

30. Pour Barthes, le texte est un exercice de style ; « (...) le seul pouvoir de l'histoire sur le 'cœur humain', c'est de varier le langage qui le parle. Tout est dit depuis plus de sept mille ans qu'il y a des hommes et qui pensent: oui, sans doute; mais on ne vient jamais trop tard pour inventer de nouveaux langages. » (Barthes, 1981: 226). Barthes moraliste comme La Bruyère car voulant comprendre le comportement collectif, et cherchant à découvrir les raisons cachées, inavouées, il pénètre dans l'univers du mythe ; et pour le mythologue sa plus grande sociabilité réside dans sa plus grande moralité. Sa liaison avec le monde est de l'ordre sarcastique (cf. Mitologias, 1973a : 222). C'est cette rupture qui lui permet de regarder à distance et d'ébranler les structures; de ne pas se contenter de décrire et vérifier comme La Bruyère, mais d'analyser et de dénoncer l'obédience inconsciemment assumée par le collectif à cet ennemi capital, la Norme bourgeoise. ( cf. Mitologias, 1973a : 3). 
31. M. Teste, le représentant de l'intellectualisme, du rigorisme, du puritanisme jusqu'à l'hyperbole, jusqu'au monstrueux ; il est celui qui ne connais le corps que dans la souffrance, qui refuse tous les plaisirs, notamment celui du texte, qui ne lit ni n'écrit. (cf. 1982 : 274).

32. À propos d'une science de la littérature, Barthes ne dit-il pas qu'« elle n'interprétera pas les symboles, mais seulement leur polyvalence » ? et que, « en un mot, son objet ne sera plus les sens pleins de l'œuvre, mais au contraire le sens vide qui les supporte tous »? (R. Barthes, 1966 : 57). 33. «L'œuvre propose, l'homme dispose. » (Barthes, 1966 : 52).

\section{RÉSUMÉS}

Le propos de cet article est de rendre compte de la réception de Roland Barthes au Portugal, entre 1973 - date de la première traduction de l'un de ses textes - et 2015. Une analyse des traductions de ses ouvrages et de leur parution à des moments charnière de la culture portugaise d'une part, et l'étude des textes critiques portant sur son œuvre de l'autre, nous permettront de dégager l'image que l'on a reçue, mais aussi celle que l'on a construite, de Roland Barthes au Portugal. Cette étude vise à montrer comment ses écrits et sa pensée furent perçus, comment il fut (re)présenté par traducteurs, éditeurs, critiques et enseignants au public portugais, plus nombreux et plus divers qu'on ne saurait le croire.

This article aims at studying the reception of Roland Barthes in Portugal, between 1973 - when one of his texts was first translated - and 2005. We will focus on the translations of his works and on how they were published at crucial moments for Portuguese culture, as well as study the critiques of his texts. This will uncover the image of Roland Barthes which was received from abroad but also constructed in Portugal. This study will show how Barthes' writings and thought were perceived, how they were (re)presented by translators, editors, critics and professors to the Portuguese public, which is wider and more diversified than one might imagine.

\section{INDEX}

Mots-clés : traduction, critique, pensée barthésienne, réception

Keywords : translation, Barthesian thought, critique, reception

\section{AUTEURS}

\section{DOMINIQUE FARIA}

CEC- Univ. Lisbonne - Universidade dos Açores

dominiquefaria@hotmail.com

\section{JOÃO DA COSTA DOMINGUES}

CLP - Universidade de Coimbra

jcosta@fl.uc.pt 